\title{
Cryo FIB Applications: Metallographic Etching of Biological Materials; Cryolithography with Ice as an Environmentally Friendly Photoresist
}

\author{
W. J. MoberlyChan*, S. Reyntjens**, A. M. Minor*** \\ *Lawrence Livermore National Laboratory, CMS-MSTD, Livermore, CA 94550-9234. \\ **FEI Inc., 5600 KA Eindhoven, The Netherlands. ***NCEM, LBL, Berkeley, CA 94720.
}

Why cryo Focused Ion Beam (FIB)? When the Gatan-Alto-2500 cryo stage is attached to the FEI-DualBeam$235 \mathrm{FIB} / \mathrm{SEM}$, all regular cryo-SEM applications are feasible, and new cryo-FIB applications are invented. In traditional cryo-SEM (e.g. hydrated flower of Fig. 1), the sample is quick-frozen, transferred to a vacuum pre-station(s), possibly fractured or sectioned by a knife, coated with a conductive film, and then imaged in the main SEM chamber. However, imaging only involves microstructure of fracture, and high magnification features can be masked by structure of the conductive coating. A more interpretable $2 \mathrm{D}$ view is provided by a cryo FIB section, with smoother finish provided by successively smaller beam sizes (20nA to 1pA), akin to successively finer grit during metallographic polishing. In a leaf stem, the (cryo) FIB-polished portion depicts no microstructure in contrast to the surrounding fracture surface (Fig. 2). As the sample warms [1], however; ice sublimes $\left(-90^{\circ} \mathrm{C}\right)$, with microstructure revealed akin to metallographic etching and imaged live by SEM. Higher magnification imaging (Fig. 2c) requires less sublimation. 3-D information is obtained by sequential images during sublimation or by successive FIB-polishing and sublimation. The DualBeams have an added advantage to neutralize each other, as shown by reduced charging in FIBed areas of Figs. 1, 4b, 5a.

Although preparations of (freeze) dry plant samples are well developed, other cells (such as blood platelets) are commonly immersed in liquid (water). Transmission imaging techniques (e.g. TEM or OM) may not be limited, but cryo SEM often sees no more than the charging top monolayers of ice. Fig. 3 exhibits features in the surrounding top frozen surface that may only be bubbles. The FIB trench can etch through the ice to reveal the cell membranes and microstructure. Microstructure can be directly visible while FIB etching [2]; and in other cases after FIB, SEM imaging during sublimation provides a series of microstructural data.

Even the lowest current FIB beam does damage. For example, Fullerene microtubules are destroyed during attempts to locate the FIB beam on their surface. Fig. 4a is an SEM image of carbon tubules (nanotubes) with metallic leads having been patterned by electron beam lithography [3]. With the sample at $-125^{\circ} \mathrm{C}$, water vapor is injected into the chamber, and ice grows on the surface as a protective coating (akin to Pt deposition or FIB-cross-section-prep). Ice deposition is monitored by SEM, with $\sim 300 \mathrm{~nm}$ growth in $\sim 15$ seconds. A 10pA FIB beam can now locate features, cut through ice, and section through a tubule. (SEM of Fig. 4b images ice coating and location of FIB-cut. The left section of the tubule is no longer visible beneath the ice because it is no longer grounded, in contrast to dark-appearing portions of tubules.) As ice sublimes away during heating (SEM image of Fig. $4 \mathrm{c}$ at $-50^{\circ} \mathrm{C}$ ), the detached portion of the tubule has also been removed.

Ice can replace photoresist to process metallization lines and pads. Ice is deposited on a $\mathrm{Si}$ wafer at $-130^{\circ} \mathrm{C}$ to a thickness of $\sim 200 \mathrm{~nm}$. The FIB cuts patterns through the ice, which etches $>100$ times faster than $\mathrm{Si}$. FIB imaging (Fig. 5a) depicts bright contrast from the Si substrate as compared to the ice. After sputter coating $\sim 30 \mathrm{~nm} \mathrm{Cr}$ onto the ice (Fig. 5b), the sample is heated to vaporize the ice. (Flakes of $\mathrm{Cr}$ persisted on the surface; however, scrubbing with a Q-tip removed the majority of excess Cr flakes.) Normal SEM imaging in Fig. 5c depicts the robustness of the metallization patterns, with line resolution $<50 \mathrm{~nm}$ achieved (although the cleaning process needs refinement to remove excess $\mathrm{Cr}$ to right in Fig. 5c).

Ref. 1 Hans Mulder, G.I.T. Imaging \& Microscopy, p.8-10, February, 2003.

Ref. 2. Julie McGeoch, submitted to Nature.

Ref 3. N. Mason, M. Biercuk, C. Marcus, Science, V303 p.655-658, (2004).

Acknowledgements: R. Schalek, J. McGeoch, R. Dickie, R. Rogers, G.King, M. Biercuk, \& CIMS @ Harvard Univ.; P. Miller, R. Wolf, \& N. Othen@ Gatan; H. Mulders, R. McDonald, \& L.Giannuzzi @ FEI; G.Ning @ Penn State Univ; $\&$ the United States Department of Energy by the University of California, LLNL under contract No. W-7405-Eng-48. 


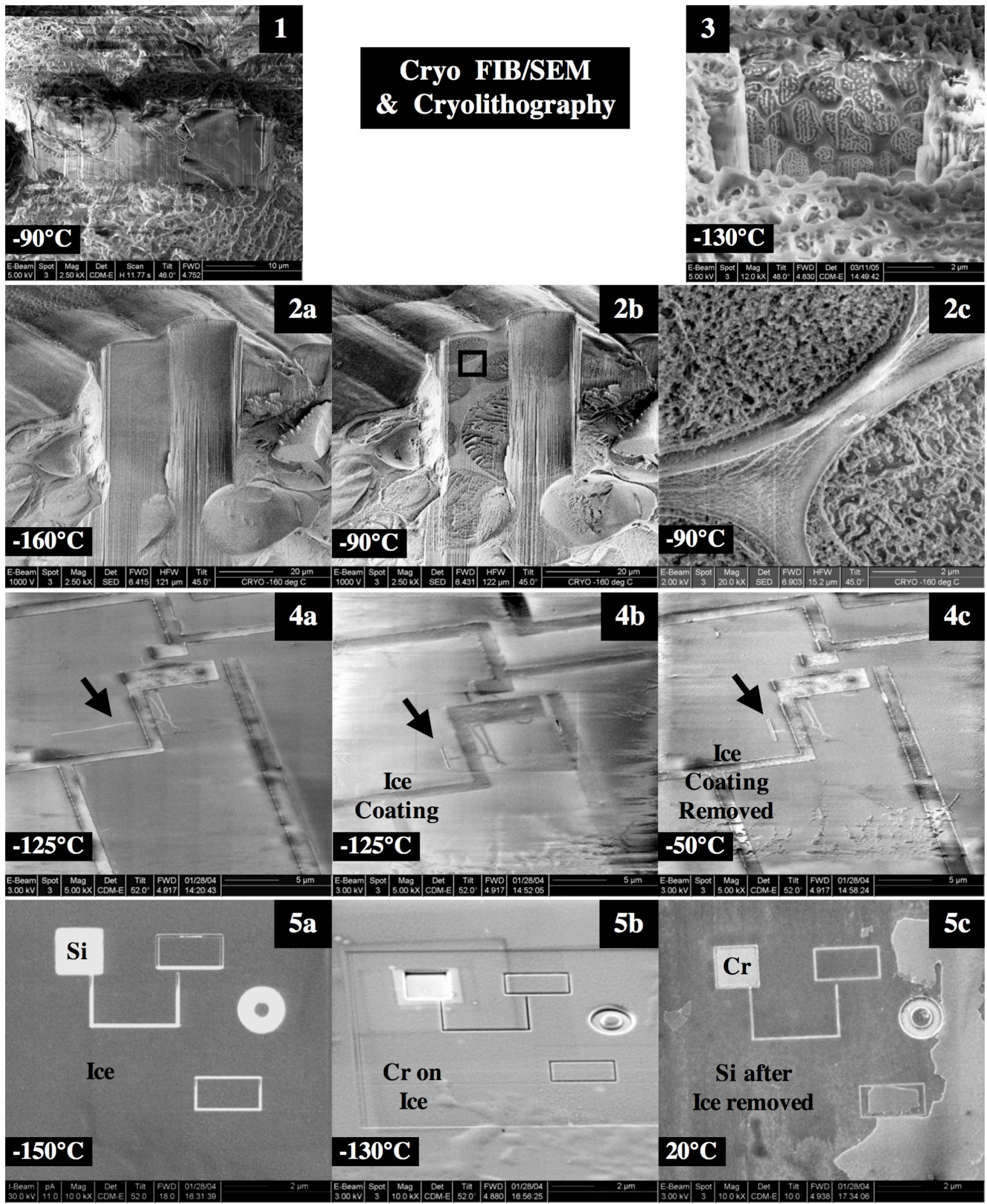

FIG. 1. SEM image of cryo-FIB-sectioned Arabidopsis (courtesy of Dr. G. Ning - Penn State). FIG. 2. SEM of leaf stem. FIG. 3. SEM of FIB-etched blood platelets in plasma (courtesy of Dr. R. Dickie \& R. Rogers of BioMedical Imaging Lab @ Harvard School of Public Health). FIG. 4. SEM of fullerene microtubules, coated with ice, FIB-cut, \& ice removed. FIG. 5. FIB through ice on $\mathrm{Si}, \mathrm{Cr}$-coated, \& ice removed. 